\title{
Macular Atrophy of the Retinal Pigment Epithelium in Patients with Neovascular Age-Related Macular Degeneration: What is the Link? Part I: A Review of Disease Characterization and Morphological Associations
}

\author{
Mania Horani (D) - Sajjad Mahmood · Tariq M. Aslam
}

Received: January 24, 2019 / Published online: March 25, 2019

(C) The Author(s) 2019

\begin{abstract}
Introduction: The purpose of this review was to explore the potential link between macular atrophy (MA) of the retinal pigment epithelium in patients with neovascular age-related macular degeneration (nAMD) with the disease characteristics and morphological features.

Methods: To this end, we performed a search of peer-reviewed articles published on the PubMed database and included all relevant papers. We then examined these papers for possible risk factors for MA development in the context of
\end{abstract}

Topical collection: Macular Atrophy of the Retinal Pigment Epithelium in Patients with Neovascular AgeRelated Macular Degeneration: What is the link?

Enhanced digital features: To view enhanced digital features for this article go to https://doi.org/10.6084/m9. figshare.7807913.

M. Horani $(\bowtie)$

Manchester Royal Eye Hospital, Manchester University Foundation NHS Trust, Manchester, UK e-mail: mania.horani@mft.nhs.uk

S. Mahmood . T. M. Aslam

Manchester Royal Eye Hospital, Manchester University Foundation NHS Trust, Manchester Academic Health Science Center, Manchester, UK

T. M. Aslam

Division of Pharmacy and Optometry, School of Health Sciences, Faculty of Biology, Medicine and Health, The University of Manchester, Manchester, UK
nAMD treated with anti-vascular endothelial growth factor drugs, as well as possible protective factors.

Results: Our review of the relevant publications revealed that areas of MA can be directly visualized through multiple imaging modalities. Associations have been identified between MA of the retinal pigment epithelium and choroidal neovascular membrane characteristics, intra- and subretinal fluid, pigment epithelial detachment, choroidal thickness, subretinal hyperreflective material, outer retinal tubulations, hemorrhage, subretinal drusenoid deposits, refractile drusen, hyperreflective foci, retinal angiomatous proliferation, polypoidal choroidal vasculopathy, geographic atrophy in the fellow eye, genetic factors, and age.

Conclusion: The findings of this review indicate that a multimodal approach is recommended for the assessment of MA. The conclusions drawn to date on the correlation between MA development or progression of MA and specific risk factors and possible protective factors are mixed. More clinical research is needed to reach a better understanding of this association.

Keywords: Anti-VEGF; Fundus autofluorescence; Macular atrophy; Neovascular agerelated macular degeneration; Optical coherence tomography 


\section{INTRODUCTION}

Age-related macular degeneration (AMD) is considered to be the leading cause of severe and irreversible visual loss in the developed world in patients aged over 55 years, and the third leading cause of blindness in the world [1,2]. While far less prevalent, the neovascular form of the disease is responsible for the overwhelming majority of serious vision loss from AMD [3].

Anti-vascular endothelial growth factor (VEGF) therapy has revolutionized the treatment of neovascular AMD (nAMD), proving to be both effective and safe in improving visual and anatomic outcomes in patients with nAMD [4-7]. In current practice, almost all patients diagnosed with nAMD are treated with antiVEGF therapy. Milestone clinical trials have demonstrated efficacy of regular intravitreal injections of the anti-VEGF drugs bevacizumab, ranibizumab, or aflibercept in terms of improving visual acuity (VA) outcomes [7-12].

Although the actual cause of vision decline in eyes on long-term treatment with VEGF inhibitors is multifactorial, there is some evidence that macular atrophy (MA) in eyes treated for nAMD is a common cause of reduced longterm visual function following initial shortterm visual gains [13-15]. In this review we examine features of macular atrophy in relation to the development and treatment of macular degeneration. We first discuss methods of MA assessment and associations with pathophysiological characteristics of macular degeneration and then we look at the association of MA with factors related to treatment of macular degeneration.

The pathogenesis of atrophic areas developing in the macula in treated nAMD is unclear and may or may not be clinically distinguishable from geographic atrophy (GA) that develops in the setting of purely non-neovascular AMD (non-nAMD). In this review, we therefore use the term 'macular atrophy' (MA) to refer to areas of atrophy within the macula of eyes with nAMD, as it serves the intended purpose without claiming that these lesions are similar to or different from GA of non-nAMD.

\section{METHOD}

We performed a search of peer-reviewed articles published on the PubMed database and included all relevant papers. We then examined possible risk factors for MA development in the context of nAMD treated with anti-VEGF drugs, as well as possible protective factors.

This review article is based on previously conducted studies and does not contain any studies with human participants or animals performed by any of the authors.

\section{DETECTION AND ASSESSMENT OF MA}

Areas of loss or attenuation of macular tissue can be directly visualized through multiple imaging modalities. It has long been accepted that total loss of retinal pigment epithelium (RPE) in areas with hypertransmission seen in spectral-domain optical coherence tomography (SD-OCT) should be regarded as evidence of atrophy [12, 16-29].

Recently, according to an international consensus, the Classification of Atrophy Meetings (CAM) group recommended that nonnAMD trials include color fundus photography (CFP), fundus autofluorescence (FAF), near-infrared reflectance (NIR), and spectral-domain or swept-source OCT [30].

Even more recently, the CAM group proposed a more detailed classification system based on OCT for AMD-related atrophy [31] in which Fourier-Domain OCT is regarded as the reference standard imaging modality to diagnose and grade atrophy. Other imaging modalities, including FAF, NIR, and color imaging, are considered to provide complementary and confirmatory information, especially in cases where OCT alone may not be sufficient for diagnostic purposes [31].

\section{Color Fundus Photography}

In CFP, MA lesions are defined as sharply demarcated areas of RPE hypopigmentation, with underlying choroidal vessels clearly visible. CFP cannot visualize many lesion characteristics associated with MA progression [32]. 


\section{Fundus Fluorescein Angiography}

With fluorescein angiography (FFA), MA is identified as well-defined areas of early hyperfluorescence termed "window defects" that are created when RPE loss enhances visualization of underlying choroidal vasculature perfused with intravascular fluorescein dye. Late staining of surrounding choroidal stromal tissue may blur MA margins in later phases of FFA, rendering the detection of MA less precise [32].

\section{Fundus Autofluorescence}

With short-wavelength FAF, MA lesions appear as well-demarcated areas of hypoautofluorescence due to the loss of RPE cells containing intrinsic fluorophores, such as lipofuscin. In a healthy retina, the fovea appears darker than the surrounding areas due to the foveal lutein; therefore evaluating foveal integrity in the presence of MA can be difficult. However using FAF in combination with NIR can enable a better foveal assessment [32, 33]. In many of the clinical trials conducted to date FAF has been widely adopted as the primary method to detect, monitor, and quantify atrophic lesions $[14,18,20-22,25,34]$.

\section{Near-Infrared Reflectance}

Near-infrared reflectance imaging uses near-infrared wavelengths that are minimally absorbed by media opacities, neurosensory layers, and macular luteal pigments. MA usually appears brighter than the surroundings, and NIR can aid in detecting foveal lesion boundaries $[33,35,36]$. However, the subfoveal choroidal thickness (SCT) can also influence signal intensity $[32,37]$.

\section{Near-Infrared Autofluorescence}

With near-infrared autofluorescence (NIA), atrophic areas appear to be hypoautofluorescent due to the lack of melanin, which is autofluorescent in the infrared spectrum and enriched in RPE cells [38]. Similar to NIR, one of the advantages of NIA is that it is unaffected by luteal pigment; however, eyes with dark irides also have strong autofluorescence from underlying choroidal melanocytes, and this signal can thus "wash out" areas of RPE loss that would otherwise appear hypoautofluorescent [38], making atrophy borders difficult to identify [34].

\section{Optical Coherence Tomography}

Based on SD-OCT, MA is defined by an abrupt increase in choroidal reflectivity below Bruch's membrane due to the loss of absorbing outer retinal structures and RPE (choroidal hypertransmission); i.e., RPE, photoreceptor, and choriocapillaris layer loss. SD-OCT has very recently been proposed by the CAM Consensus as a reference standard imaging modality to diagnose and grade atrophy [31]. Recognizing that atrophy can evolve to different stages, the CAM Consensus suggests four histologic terms: complete RPE and outer retinal atrophy (cRORA); incomplete RPE and outer retinal atrophy (iRORA); complete outer retinal atrophy (CORA); and incomplete outer retinal atrophy (iORA). cRORA is defined as a zone of homogeneous choroidal hypertransmission and absence of the RPE band measuring $\geq 250 \mu \mathrm{m}$ with overlying outer retinal thinning and photoreceptor loss, in the absence of an RPE tear. iRORA is characterized by discontinuous choroidal hypertransmission, RPE band and photoreceptor loss, in cases where the criteria for cRORA are not met. In cORA, hypertransmission is only intermittent, yet there is a continuous absence of ellipsoid zone with severe thinning of the outer retina, in the setting of a largely intact RPE band. iORA demonstrates a detectable ellipsoid disruption, thinning of the outer retina, an intact RPE band, and no hypertransmission [31].

\section{OCT Angiography}

Optical coherence tomography-angiography (OCT-A) detects blood flow by analyzing the changes in tissue reflectivity that occur between rapidly acquired images, enabling three- 
dimensional reconstruction of retinal and choroidal vasculature. Absence of choriocapillaris flow within a MA lesion has been reported with OCT-A. Alterations in choriocapillaris flow outside the GA lesion are also apparent in some eyes with MA [32, 39-41].

\section{Methods of MA Detection and Assessment in Clinical Studies}

Numerous clinical studies (see following list) have worked towards achieving a better understanding of MA. However, the reports based on these studies have used various terms to describe what is roughly the common subject of interest in this review, i.e., areas of atrophy within the macula of eyes with nAMD. The term GA has been applied inconsistently, especially in papers recording changes in atrophy related to wet AMD and its treatment.

The reviewed clinical studies have also used relatively different inclusion criteria and have relied on different imaging modalities to detect and assess MA.

These studies include:

The Comparison of Age-related Macular Degeneration Treatments Trials (CATT) study and HARBOR study, both of which relied on CFP and/or FFA to detect atrophy of the macula [13, 42-48]. In comparison, the authors of the Inhibition of VEGF in Agerelated choroidal Neovascularization (IVAN) study, Tanaka et al., and Kuroda et al. utilized OCT in addition to CFP and FFA for atrophy assessment [12, 16, 19, 24].

Zarubina et al. [23], $\mathrm{Xu}$ et al. [17] , and the authors of the TREX-AMD study all assessed areas of atrophy using NIR and SD-OCT.

In the TREX-AMD study, MA was mainly detected and quantified using FAF [20, 21]. In the SEVEN-UP study, MA was detected using FAF [14, 34]. In their study, Thavikulwat et al. [25] relied on several modalities, including FAF, CFP, FFA, and OCT [25]. Similarly, Hata et al. [22] evaluated MA using CFP, SD-OCT, and FAF [22].

Schütze et al. [18] used a polarization-sensitive OCT segmentation algorithm and correlated the algorithm with SD-OCT and FAF findings.

Young et al. [28] and Wons et al. [29] determined the RPE atrophy area using OCT.

Table 1 shows and summarizes the imaging modalities and criteria used in detecting and assessing MA in the relevant studies on nAMD eyes treated with anti-VEGF drugs.

\section{PHENOTYPE CHARACTERISTICS ASSOCIATED WITH MACULAR ATROPHY}

Several studies have identified a correlation between MA and particular factors. Some factors have been found to increase the risk of developing MA or its progression, as noted in Table 2 . Alternatively, others were found to provide a protective effect from MA, as reported in Table 3.

\section{Choroidal Neovascularization Characteristics and MA}

Tanaka et al. [16] suggested that during the course of anti-VEGF therapy GA typically does not progress beyond the original boundaries of a choroidal neovascularization (CNV) lesion unless the eye had GA outside these boundaries at the time anti-VEGF treatment was initiated. This suggestion was based on the results of their retrospective cohort study of 75 patients with a follow-up of at least 3.5 years after the start of anti-VEGF therapy [16].

Eyes that demonstrate enlargement of GA outside the original boundaries of the $\mathrm{CNV}$ lesion appear to be eyes manifesting enlargement of preexisting GA that is likely unrelated to the $\mathrm{CNV}$, similar to how GA typically enlarges in eyes with drusen in the absence of $\mathrm{CNV}$ or anti-VEGF therapy [16].

A retrospective study by $\mathrm{Xu}$ et al. [17] of 94 eyes on anti-VEGF therapy for a minimum of 12 months found that $55.3 \%$ of eyes experienced apparent GA growth. The neovascularization type was a major factor related to GA development. The odds of developing apparent GA were significantly lower in type 1 


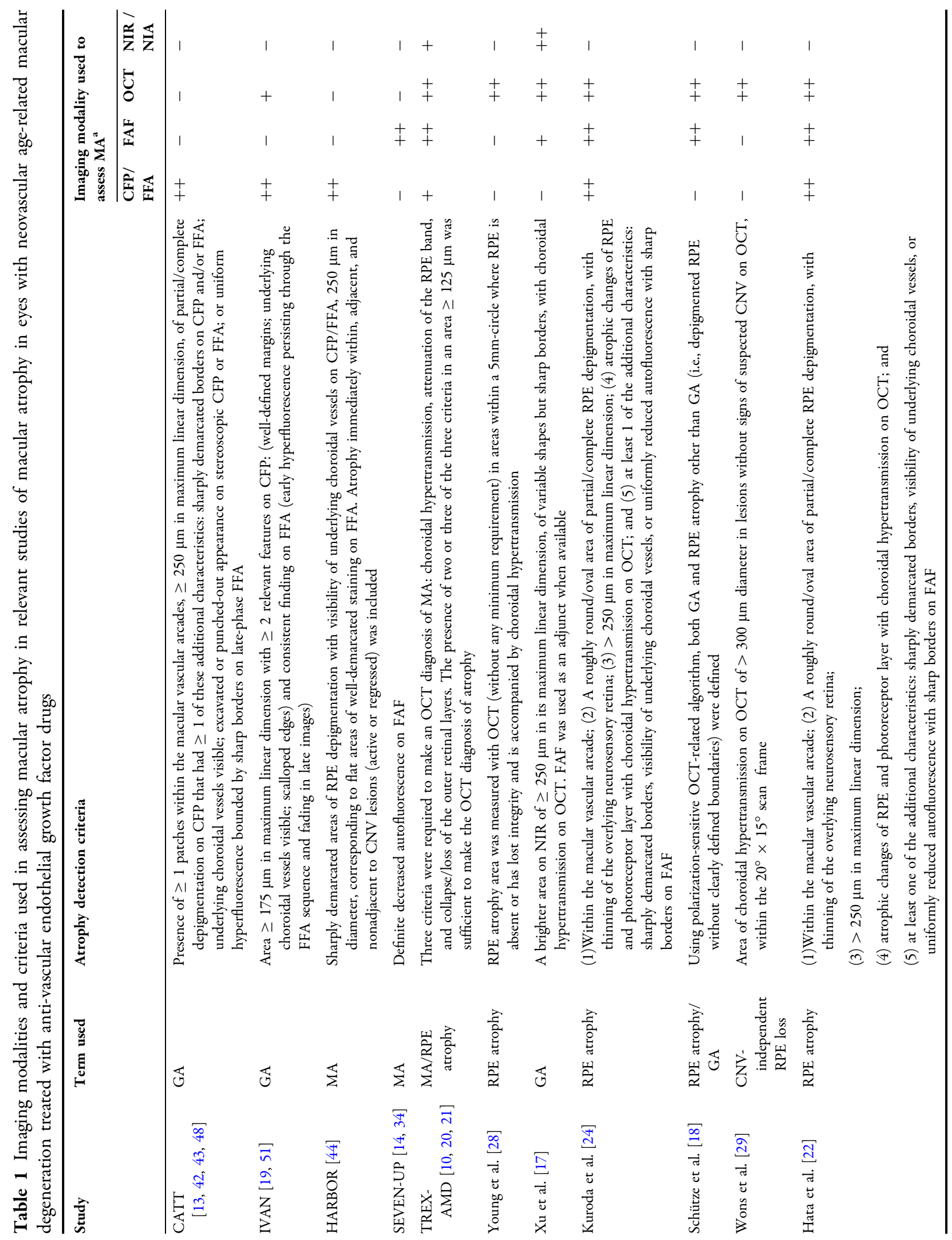




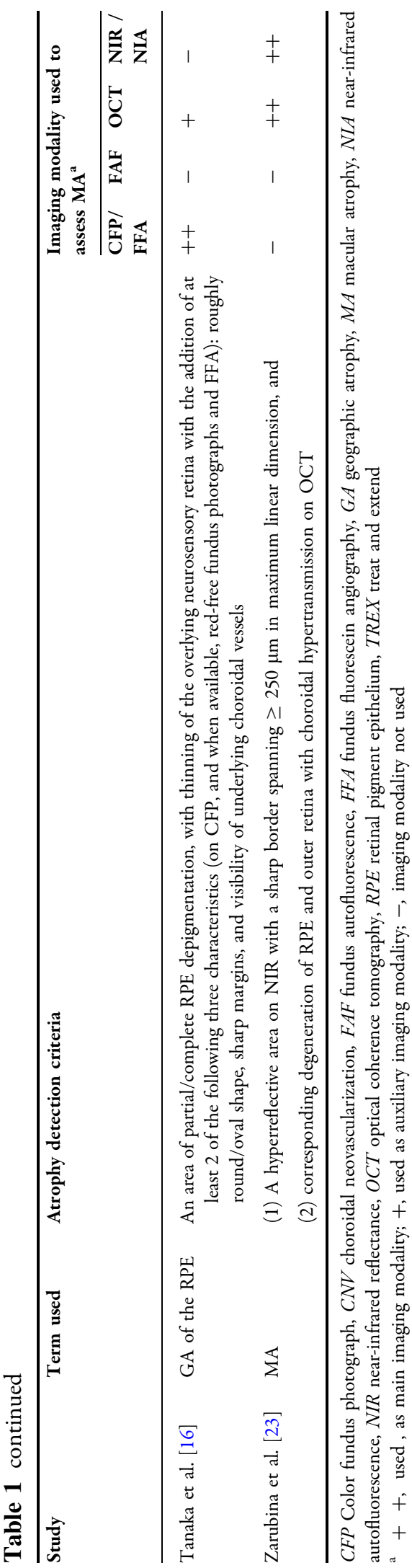

neovascularization compared to the other lesion types [17]. The authors stressed the importance of the anatomical classification of $\mathrm{CNV}$, as the CNV type affected the prognosis of GA development in eyes treated with anti-VEGF therapy for nAMD [17] because eyes with type I CNV (occult) had been previously found to be resistant to the development of GA [49]. This finding by $\mathrm{Xu}$ et al. was supported by previous studies; for example, Grossniklaus and Green [50] suggested that the RPE and the photoreceptors obtain some nutritional support from the neovascular tissue underneath the RPE in type I CNV.

In the CATT study, the baseline CNV size correlated with the risk for GA development, so that a larger $\mathrm{CNV}$ area at baseline was a risk factor for GA development [42]. At 2 years of follow-up, results from the CATT study also indicated that eccentricity from the central fovea and more classic component of CNV were associated with higher GA growth rates [13]; these results were confirmed again in the 5-year report [43].

\section{Intraretinal Fluid and MA}

Intraretinal fluid at the foveal center was one of the baseline risk factors found for GA development in the CATT study [42].

Post hoc analysis of the HARBOR study results found that development of MA was associated with baseline presence of intraretinal cysts [44]. Interestingly, Schütze et al. [18] reported that localized RPE atrophy was frequently observed beneath intraretinal cysts and that fluid accumulation was present, associated with the dysfunctional neurosensory retina.

\section{Subretinal Fluid and MA}

The authors of the CATT study reported that favorable baseline factors associated with a lower risk for GA development included OCT measurements of subretinal fluid (SRF) thickness of $>25 \mu \mathrm{m}$ [13].

In the HARBOR trial, some patients with residual CNV activity and small amounts of SRF had less MA $[45,46]$. The authors of this trial 


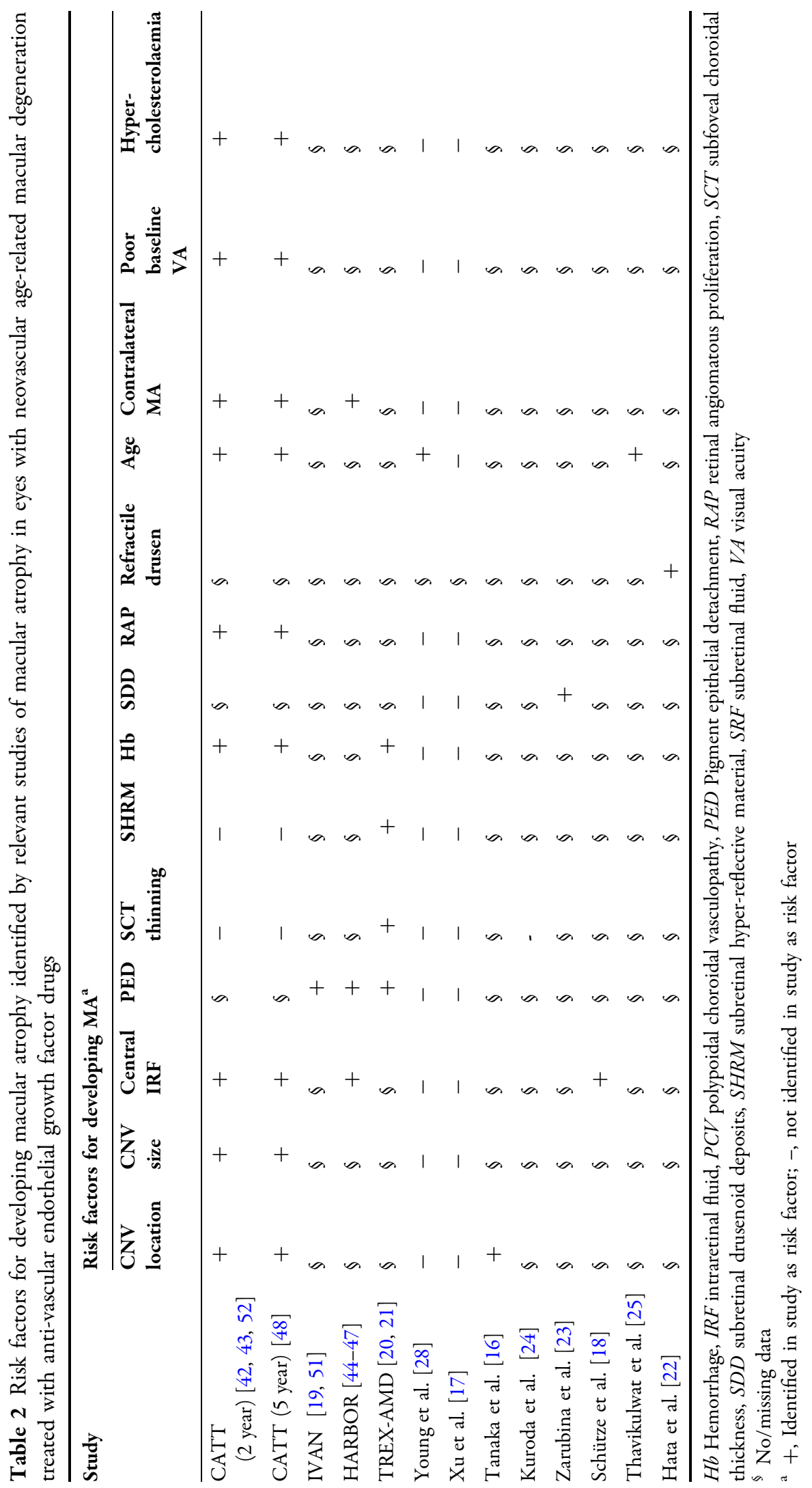


also reported that baseline SRF presence was inversely associated with MA [44].

\section{Pigment Epithelial Detachment and MA}

The authors of the IVAN study reported that 12 of the 18 eyes with pigment epithelial detachment (PED) at baseline that were treated with anti-VEGF therapy and which showed GA at a follow-up visit, also showed the area of GA to be localized to the site of PED. The strong spatial localization of GA to the area of pre-existing PED suggests a predisposition to GA in eyes with this characteristic $[19,51]$.

A post hoc analysis of the HARBOR study results also suggested an association between PED and MA. Among the study eyes with PED at baseline but no detectable MA at baseline, there was an approximately threefold higher rate of MA development in patients with complete flattening of PED compared with patients with persistent PED at month 24. MA development at month 24 was independent of PED size at baseline [45-47]. These data support a concept that treatment to resolve PED completely may not be essential as more MA is seen when the PED is resolved completely. However, it is unclear from the data whether MA preceded or followed PED resolution, nor has the type of PED been specified in this study. Also, these data are the result of an exploratory post hoc subgroup analysis and the results should be interpreted with caution [47].

In the TREX-AMD study, PED thickness was a significant predictor of new MA development in eyes with no MA at baseline [20].

In the CATT study, a sub-RPE tissue complex thickness of $>275 \mu \mathrm{m}$ was associated with a lower risk for GA development [13]. In addition, absence of sub-RPE fluid at baseline was found to be associated with a higher growth rate [43].

\section{Subfoveal Choroidal Thickness and MA}

In the TREX-AMD study, subfoveal choroidal thickness (SCT) was measured at baseline with SD-OCT using the electronic caliper available on the software. Subfoveal choroidal thickness was measured at a single point in the foveal center and defined as the distance from the external edge of the RPE to the choroidoscleral junction [20]. The baseline SCT was correlated with the presence of MA at baseline and the development of incident MA by month 18 [21]. Baseline SCT in eyes with MA was statistically significantly less than in those eyes without MA in both the non-nAMD and nAMD groups. The cutoff threshold of SCT for predicting the development of MA in cases without MA at baseline was $124 \mu \mathrm{m}$. Among eyes without MA at baseline, those with a baseline SCT of $\leq 124 \mu \mathrm{m}$ were 4 .3-fold more likely to develop MA than those with baseline SCT of $>124 \mu \mathrm{m}$ [21]. Eyes with a thinner SCT at baseline tended to have more MA at month 18 and vice versa.

A study by Hata et al. [22] found that at 12 months of treatment of eyes with retinal angiomatous proliferation (RAP), the progression rate of RPE atrophy area was negatively correlated with baseline SCT.

\section{Subretinal Hyper-Reflective Material and MA}

In nAMD, subretinal hyper-reflective material (SHRM) is a morphological feature seen on OCT as hyper-reflective material located external to the retina and internal to the RPE. Willoughby et al. [52] found a strong correlation between the presence of SHRM and scar formation.

In the TREX-AMD study, the presence of baseline SHRM and also baseline SHRM thickness were significant predictors of new MA development in eyes with no baseline MA. Eyes with a thicker SHRM at baseline tended to have more MA at month 18 and vice versa [20].

\section{Outer Retinal Tubulations and MA}

Further work [52] on eyes in the CATT study was conducted to analyze a subset of eyes that were imaged with SD-OCT beginning at week 56 and again at week 104. Poor VA, greater lesion size, and presence of baseline SHRM were associated independently of each other with greater risk of outer retinal tubulations (ORT). The same factors were also identified as risk factors for developing 
Table 3 Protective factors from developing macular atrophy identified by relevant studies of macular atrophy in eyes with neovascular age-related macular degeneration treated with anti-vascular endothelial growth factor drugs

\begin{tabular}{lllllll}
\hline Study & \multicolumn{7}{l}{ Protective factors from developing $\mathbf{M A}^{\mathbf{a}}$} & \\
\cline { 2 - 7 } & $\begin{array}{l}\text { Type 1 } \\
\text { CNV }\end{array}$ & $\begin{array}{l}\text { Blocked } \\
\text { fluorescence }\end{array}$ & SRF & PCV & $\begin{array}{l}\text { Sub-RPE complex } \\
\text { thickness }\end{array}$ & $\begin{array}{l}\text { Vitromacular } \\
\text { attachment }\end{array}$ \\
\hline CATT (2 year) [42, 43] & ++ & ++ & ++ & $\wp$ & ++ & ++ \\
CATT (5 year) [48] & ++ & + & ++ & $\S$ & ++ & + \\
HARBOR [44-46] & $\S$ & $\S$ & ++ & $\S$ & $\S$ & $\S$ \\
Xu et al. [17] & ++ & $\S$ & $\S$ & $\S$ & $\S$ & $\S$ \\
Kuroda et al. [24] & $\S$ & $\S$ & $\S$ & ++ & $\S$ & $\S$ \\
\hline
\end{tabular}

$\checkmark$ No/missing data

${ }^{\text {a }}++$, identified in study as significant protective factor; + , identified in study as non-significant protective factor

MA itself [21, 53]. Furthermore, GA was found to be itself a risk factor for ORT [53].

The aforementioned apparent agreement seen in the CATT study between the risk factors identified for MA and those identified for ORT might create an impression that MA and ORT are correlated. However, the MAHALO study, a cohort study on fellow untreated eyes within the CATT study which examined the effect of lampalizumab in patients with GA associated with nonnAMD, has proven the opposite [54]. In MAHALO, 108 baseline and month 18 data of fellow eyes were studied. The enlargement rate of GA in eyes with ORT was found to be significantly slower than that of eyes without ORT [54].

Finally, the absence of diabetes and blocked fluorescence have both been found to increase the risk for ORT. However, conversely, in the CATT study, blocked fluorescence was identified as a favorable factor that provided protection from MA [53].

Similar confusing conflictions have been shown in dry AMD studies as well, where the presence [55] and absence [56] of ORT were both reported with greater GA enlargement rates. Further Studies are needed to provide convincing explanations.

\section{Hemorrhage and MA}

In the TREX-AMD study, the presence of baseline hemorrhage was a significant predictor of new MA development in eyes with no baseline MA [20].
In the CATT study, hemorrhage at baseline was found to be associated with a higher MA growth rate [43].

\section{Subretinal Drusenoid Deposits and MA}

Zarubina et al. [23] looked back at data from 74 eyes without pre-existing MA receiving antiVEGF therapy for nAMD over a mean follow-up of 4.7 years on a continuous treat-and-extend regimen. Of these 74 eyes, 51\% developed MA. Compared with eyes without subretinal drusenoid deposits (SDD), those with SDD at baseline were threefold more likely to develop MA. Eyes with SDD present in the inferior macula and inferior extramacular fields at baseline were three- and 6.5-fold more likely to develop MA at follow-up than eyes without SDD in these locations. SDD were independently associated with MA development [23].

\section{Retinal Angiomatous Proliferation and MA}

Retinal angiomatous proliferation (RAP) was identified in the CATT study as one of the baseline risk factors for GA development [42]. A study by Hata et al. [22] included 46 eyes with treatment-naive RAP. The progression rate of RPE atrophy area was negatively correlated with baseline SCT. RPE atrophy developed in 36.6\% 
of the eyes during the 12 months following anti-VEGF treatment for RAP [22].

\section{Polypoidal Choroidal Vasculopathy and MA}

Kuroda et al. [24] reported the results from their retrospective interventional case series that included 195 eyes with treatment-naïve subfoveal nAMD treated as-needed (PRN) after three once-monthly treatments with ranibizumab. Polypoidal choroidal vasculopathy (PCV) was associated with a lower incidence and a slower progression of RPE atrophy compared to typical nAMD [24].

\section{Refractile Drusen and MA}

Hata et al. [22] reported refractile drusen in RAP eyes at baseline to be associated with RPE atrophy development at 12 months. These authors identified the presence of refractile drusen at baseline as a novel significant risk factor for RPE atrophy development [22].

\section{Hyperreflective Foci and MA}

Hyperreflective foci (HRF) are regarded as a predictor of $\mathrm{CNV}$ and leading hallmark of GA.

HRF are discrete, well-circumscribed lesions with a greater reflectivity than the RPE band on SDOCT [57]. HRF overlying drusen are likely to represent disease progression, with RPE migration into the retina and possible photoreceptor degeneration or glial scar formation [58]. It has been reported that proliferation and inner retinal migration of HRF occurred during the follow-up in eyes with intermediate AMD and that these were associated with a higher incidence of GA $[59,60]$.

\section{Contralateral GA}

In the CATT study, GA in the fellow eye was considered to be a baseline risk factor for GA development [42]. Furthermore, at 2 years of follow-up, the growth rate of GA was higher for eyes with contralateral GA [13]. The same was still true at 5 years [43].
Post hoc analysis of the HARBOR study found that development of MA was associated with MA in the fellow eye at baseline [44].

\section{MA Location}

In the CATT study, GA was reported to be nonfoveal through the 2-year initial period of the study [42]. On the contrary, in the SEVEN-UP study, MA was foveal-involving in $90 \%$ of study eyes [14]. Schütze et al. [18] reported that the focal RPE lesions detected were diffusely distributed and not exclusively located subfoveally.

\section{MA Enlargement Rate}

The change in total MA lesion area over time $\left(\mathrm{mm}^{2} /\right.$ year $)$ is the most frequently used and accepted endpoint for assessing MA progression, and the value of this endpoint is usually presented after square-root transformation (mm/year). Significant predictors of the final area of RPE loss includes the duration of time since baseline and the square root of the area of baseline RPE loss [61]. Eyes with baseline GA have a greater enlargement rate compared to eyes developing new-onset GA [25]. GA in the fellow eye, hemorrhage, epiretinal membrane, eccentricity from central fovea, more classic component of $\mathrm{CNV}$, and absence of sub-RPE fluid at baseline have all been associated with a higher growth rate [43].

\section{OTHER RELEVANT FACTORS AND MA}

\section{Genetic Risk Factors}

At 2 years, the CATT study found that there were no strong associations between the development of GA and the presence of risk alleles for the $\mathrm{CFH}$ (complement factor $\mathrm{H}$ ), ARMS2 (age-related maculopathy susceptibility 2), HTRA1 (HtrA serine peptidase 1), C3 (complement C3), or TLR3 (Tolllike receptor 3) genes [13].

Kuroda et al. [24] reported that the ARMS2 and $\mathrm{CFH}$ polymorphisms were significantly associated with baseline RPE atrophy, and that the $\mathrm{G}$ allele of ARMS2 A69S and the A allele of $C F H \mathrm{I} 62 \mathrm{~V}$ were risk 
alleles for baseline atrophy [24], although their analysis of genetic association did not show strong effects of the ARMS2/HTRA1 and the CFH gene polymorphisms in the development of RPE atrophy. Moreover, their data implies that AMD-associated single nucleotide polymorphisms (SNPs) have no influence on atrophy growth rates [24].

In their cohort of RAP cases, Hata et al. [22] concluded that gene polymorphisms were not associated with RPE atrophy. They did not find any significant associations of two RAP-associated SNPs in ARMS2 and CFH with the development or the growth rate of RPE atrophy in RAP receiving anti-VEGF therapy [22].

\section{Age}

Thavikulwat et al. [25] reported that increasing age is a baseline risk factor for GA development; this association was also observed in the CATT study [42].

\section{Other Factors}

The authors of the CATT study reported that hypercholesterolemia and poor baseline VA are baseline risk factors for GA development. Also, in CATT, at 2 years of follow-up, the growth rate of GA was higher for eyes with epiretinal membrane [13]; the same still applied at 5 years [43].

On the other hand, favorable baseline factors associated with lower risk for GA development at 2 years in the CATT study included blocked fluorescence and vitreomacular attachment [42]. However, these factors were no longer statistically significantly associated with risk of GA at 5 years $[43,48]$.

\section{DISCUSSION}

Despite the advances in the treatments available for nAMD, nAMD-related MA remains irreversible. In the SEVEN-UP study, about half of the nAMD patients suffered significant vision loss at the end of 7 years, mainly due to MA. MA is reported to be the most prominent chronic factor determining long-term vision in eyes treated for nAMD $[14,34]$. Both the presence of subfoveal
MA and increased area of MA are associated with decreased best-corrected VA, with the latter being the factor correlating most strongly with poor long-term visual outcome [34].

Research to date shows an expansion of atrophy during anti-VEGF treatment, but the conclusions drawn on the correlation of MA development or progression with particular risk factors and possible protective factors are mixed (see Tables 2 and 3).

Not all of the relevant clinical studies included in this review were of the same size or design; consequently they provide different levels of evidence. The studies had relatively different inclusion criteria and relied on different imaging modalities. Several studies, of significant weight, lacked FAF imaging modality, while a number of other large studies did not utilize OCT to detect, assess or monitor MA $[42-48,62,63]$. The reports of the studies therefore should be interpreted with caution.

FAF is the most widely used imaging modality in MA studies, based on the current literature; however, it is likely that use of OCT will increase still further in the future. The accuracy of the OCT-derived layer-by-layer information and its superior value in detecting, monitoring, and quantifying MA lesions should not encourage the disuse of FAF as it still has a well-established role. A multimodal approach consisting at least of OCT and FAF remains the optimal choice and recommended method.

The development of prevention and possible treatment strategies for MA will benefit from a better understanding of its pathophysiology and risk factors. More clinical research is still needed to further understand this association.

\section{ACKNOWLEDGMENTS}

Funding. No funding or sponsorship was received for this study or publication of this article.

Authorship. All named authors meet the International Committee of Medical Journal Editors (ICMJE) criteria for authorship for this article, take responsibility for the integrity of 
the work as a whole, and have given their approval for this version to be published.

Disclosures. Mania Horani and Sajjad Mahmood have nothing to disclose. Tariq M. Aslam is the European Editor-in-Chief of Ophthalmology and Therapy.

Compliance with Ethics Guidelines. This article is based on previously conducted studies and does not contain any studies with human participants or animals performed by any of the authors.

Data Availability. Data sharing is not applicable to this article as no datasets were generated or analyzed during the current study.

Open Access. This article is distributed under the terms of the Creative Commons Attribution-NonCommercial 4.0 International License (http://creativecommons.org/licenses/ by-nc/4.0/), which permits any noncommercial use, distribution, and reproduction in any medium, provided you give appropriate credit to the original author(s) and the source, provide a link to the Creative Commons license, and indicate if changes were made.

\section{REFERENCES}

1. Pedrosa AC, Sousa T, Pinheiro-Costa J, et al. Treatment of neovascular age-related macular degeneration with anti-VEGF agents: predictive factors of long-term visual outcomes. J Ophthalmol. 2017;2017:4263017.

2. Tah V, Orlans HO, Hyer J, et al. Anti-VEGF therapy and the retina: an update. J Ophthalmol. 2015;2015:627674.

3. American Academy of Ophthalmology Retina/ Vitreous Panel. Preferred practice pattern guidelines. Age-related macular degeneration. San Francisco: American Academy of Ophthalmology; 2015

4. Agarwal A, Rhoades WR, Hanout M, et al. Management of neovascular age-related macular degeneration: Current state-of-the-art care for optimizing visual outcomes and therapies in development. Clin Ophthalmol. 2015;9:1001-155.
5. Nguyen DH, Luo J, Zhang K, Zhang M. Current therapeutic approaches in neovascular age-related macular degeneration. Discov Med. 2013;15:343-8.

6. Solomon SD, Lindsley K, Vedula SS, Krzystolik MG, Hawkins BS. Anti-vascular endothelial growth factor for neovascular age-related macular degeneration. Cochrane Database Syst Rev. 2014;8:CD005139.

7. Heier JS, Brown DM, Chong V, Korobelnik JF, Kaiser PK, Nguyen QD, et al. Intravitreal aflibercept (VEGF trap-eye) in wet age-related macular degeneration. Ophthalmology. 2012;119:2537-48.

8. Brown DM, Kaiser PK, Michels M, et al. Ranibizumab versus verteporfin for neovascular age-related macular degeneration. $\mathrm{N}$ Engl J Med. 2006;355:1432-44.

9. Brown DM, Michels M, Kaiser PK, et al. Ranibizumab versus verteporfin photodynamic therapy for neovascular age-related macular degeneration: twoyear results of the ANCHOR study. Ophthalmology. 2009;116:57-65.e5.

10. Wykoff CC, Croft DE, Brown DM, et al. Prospective trial of treat-and-extend versus monthly dosing for neovascular age-related macular degeneration: TREX-AMD 1-year results. Ophthalmology. 2015;122:2514-22.

11. Chakravarthy U, Harding SP, Rogers CA, et al. Ranibizumab versus bevacizumab to treat neovascular age-related macular degeneration: One-year findings from the IVAN randomized trial. Ophthalmology. 2012;119:1399-411.

12. Chakravarthy U, Harding SP, Rogers CA, et al. Alternative treatments to inhibit VEGF in age-related choroidal neovascularisation: 2-year findings of the IVAN randomised controlled trial. Lancet. 2013;382:1258-67.

13. Grunwald JE, Daniel E, Huang J, et al. Risk of geographic atrophy in the comparison of age-related macular degeneration treatments trials. Ophthalmology. 2014;121:150-61.

14. Rofagha S, Bhisitkul RB, Boyer DS, Sadda SR, Zhang $\mathrm{K}$, Seven-Up Study Group. Seven-year outcomes in ranibizumab-treated patients in ANCHOR, MARINA and HORIZON: a multicenter cohort study (SEVENUP) Ophthalmology. 2013;120(11):2292-2299.

15. Bhisitkul RB, Desai SJ, Boyer DS, Sadda SR, Zhang K. Fellow eye comparisons for 7-year outcomes in ranibizumab-treated AMD subjects from ANCHOR, MARINA, and HORIZON (SEVEN-UP Study). Ophthalmology. 2016;123:1269-77. 
16. Tanaka E, Chaikitmongkol V, Bressler SB, Bressler NM. Vision-threatening lesions developing with longer-term follow-up after treatment of neovascular age-related macular degeneration. Ophthalmology. 2015;122:153-61.

17. Xu L, Mrejen S, Jung JJ, et al. Geographic atrophy in patients receiving anti-vascular endothelial growth factor for neovascular age-related macular degeneration. Retina. 2015;35:176-86.

18. Schütze C, Wedl $M$, Baumann B, Pircher $M$, Hitzenberger CK, Schmidt-Erfurth U. Progression of retinal pigment epithelial atrophy in antiangiogenic therapy of neovascular age-related macular degeneration. Am J Ophthalmol. 2015;159(6):1100-14.

19. Chakravarthy U, Harding SP, Rogers CA, et al. A randomised controlled trial to assess the clinical effectiveness and cost-effectiveness of alternative treatments to inhibit VEGF in age-related choroidal Neovascularisation (IVAN). Health Technology Assessment, No. 19.78. Southampton: NIHR Journals Library; 2015.

20. Abdelfattah NS, Al-Sheikh M, Pitetta S, et al. Macular atrophy in neovascular age-related macular degeneration with monthly versus treat-and-extend ranibizumab: findings from the TREX-AMD trial. Ophthalmology. 2017;124:215-23.

21. Fan W, Abdelfattah NS, Uji A, et al. Subfoveal choroidal thickness predicts macular atrophy in age-related macular degeneration: results from the TREX-AMD trial. Graefes Arch Clin Exp Ophthalmol. 2018;256(3):511-8.

22. Hata M, Yamashiro K, Oishi A, et al. Retinal pigment epithelial atrophy after anti-vascular endothelial growth factor injections for retinal angiomatous proliferation. Retina. 2017;37(11):2069-2077.

23. Zarubina AV, Gal-Or O, Huisingh CE, Owsley C, Freund KB. Macular atrophy development and subretinal drusenoid deposits in anti-vascular endothelial growth factor treated age-related macular degeneration. Invest Ophthalmol Vis Sci. 2017;58(14):6038-6045.

24. Kuroda Y, Yamashiro K, Tsujikawa A, et al. Retinal pigment epithelial atrophy in neovascular age-related macular degeneration after ranibizumab treatment. Am J Ophthalmol. 2016;161:94-103.

25. Thavikulwat AT, Jacobs-El N, Kim JS, et al. Evolution of geographic atrophy in participants treated with ranibizumab for neovascular age related macular degeneration. Ophthalmol Retina. 2017;1(1):34-41.
26. Holz FG, Bindewald-Wittich A, Fleckenstein M, et al. Progression of geographic atrophy and impact of fundus autofluorescence patterns in age-related macular degeneration. Am J Ophthalmol. 2007; 143:463-472.

27. Sunness JS, Margalit E, Srikumaran D, et al. The longterm natural history of geographic atrophy from agerelated macular degeneration. Enlargement of atrophy and implications for interventional clinical trials. Ophthalmology. 2007;114:271-277.

28. Young M, Chui L, Fallah N, et al. Exacerbation of choroidal and retinal pigment epithelial atrophy after anti-vascular endothelial growth factor treatment in neovascular age-related macular degeneration. Retina. 2014;34:1308-15.

29. Wons J, Wirth MA, Graf N, Becker MD, Michels S. Comparison of progression rate of retinal pigment epithelium loss in patients with neovascular agerelated macular degeneration treated with ranibizumab and aflibercept. J Ophthalmol. $2017 ; 7432739$.

30. Holz FG, Sadda SR, Staurenghi G, et al. Imaging protocols in clinical studies in advanced age-related macular degeneration: recommendations from classification of atrophy consensus meetings Ophthalmology. 2017;124:464-478

31. Sadda SR, Guymer R, Holz FG, et al. Consensus definition for atrophy associated with age-related macular degeneration on OCT: classification of atrophy report 3. Ophthalmology. 2017;125(4):537-48.

32. Fleckenstein M, Mitchell P, Freund KB, et al. The progression of geographic atrophy secondary to age-related macular degeneration. Ophthalmology. 2018;125(3):369-90.

33. Lindner M, Boker A, Mauschitz MM, et al. Directional kinetics of geographic atrophy progression in age-related macular degeneration with foveal sparing. Ophthalmology. 2015;122:1356-65.

34. Bhisitkul RB, Mendes TS, Rofagha S, et al. Macular atrophy progression and 7-year vision outcomes in subjects from the anchor, marina, and horizon studies: the seven-up study. Am J Ophthalmol. 2015;159(5):915-24.

35. Gobel AP, Fleckenstein M, Schmitz-Valckenberg S, et al. Imaging geographic atrophy in age-related macular degeneration. Ophthalmologica. 2011;226:182-90.

36. Holz FG, Steinberg JS, Gobel A, et al. Fundus autofluorescence imaging in dry AMD: 2014 Jules Gonin lecture of the Retina Research Foundation. Graefes Arch Clin Exp Ophthalmol. 2015;253:7-16 
37. Dolz-Marco R, Gal-Or O, Freund KB. Choroidal thickness influences near-infrared reflectance intensity in eyes with geographic atrophy due to age-related macular degeneration. Invest Ophthalmol Vis Sci. 2016;57:6440-6.

38. Keilhauer CN, Delori FC. Near-infrared autofluorescence imaging of the fundus: visualization of ocular melanin. Invest Ophthalmol Vis Sci. 2006;47:3556-644.

39. Waheed NK, Moult EM, Fujimoto JG, Rosenfeld PJ. Optical coherence tomography angiography of dry age-related macular degeneration. Dev Ophthalmol. 2016;56:91-100.

40. Kvanta A, Casselholm de Salles M, Amren U, Bartuma H. Optical coherence tomography angiography of the foveal microvasculature in geographic atrophy. Retina. 2017;37:936-942.

41. Pellegrini M, Acquistapace A, Oldani M, et al. Dark atrophy: an optical coherence tomography angiography study. Ophthalmology. 2016;123:1879-86.

42. Comparison of Age-related Macular Degeneration Treatments Trials (CATT) Research Group, Martin DF, Maguire MG, et al. Ranibizumab and bevacizumab for treatment of neovascular age-related macular degeneration: two-year results. Ophthalmology. 2012; 119(7):1388-98.

43. Grunwald JE, Pistilli M, Ying GS, et al. Growth of geographic atrophy in the comparison of age-related macular degeneration treatments trials. Ophthalmology. 2015;122:809-16.

44. Holz FG, Tuomi L, Ding B, Hopkins JJ. Development of atrophy in neovascular AMD treated with ranibizumab in the HARBOR study. Invest Ophthalmol Vis Sci. 2015;56 (7) (ARVO E- Abstract 890).

45. Busbee BG, Ho AC, Brown DM, et al.. Twelvemonth efficacy and safety of $0.5 \mathrm{mg}$ or $2.0 \mathrm{mg}$ ranibizumab in patients with subfoveal neovascular age-related macular degeneration. Ophthalmology. 2013;120(5):1046-56.

46. Ho AC, Busbee BG, Regillo CD, et al.. Twenty-fourmonth efficacy and safety of $0.5 \mathrm{mg}$ or $2.0 \mathrm{mg}$ ranibizumab in patients with subfoveal neovascular age-related macular degeneration. Ophthalmology. 2014;121(11):2181-92.

47. Sarraf D, London NJS, Khurana RN, et al. Ranibizumab treatment for pigment epithelial detachment secondary to neovascular age-related macular degeneration post hoc analysis of the HARBOR study. Ophthalmology. 2016;123:2213-24.

48. Comparison of Age-related Macular Degeneration Treatments Trials (CATT) Research Group, Maguire
MG, Martin DF, et al. Five-year outcomes with antivascular endothelial growth factor treatment of neovascular age-related macular degeneration: the comparison of age-related macular degeneration treatments trials. Ophthalmology. 2016;123(8):1751-61.

49. Engelbert M, Zweifel SA, Freund KB. Long-term follow-up for type 1 (subretinal pigment epithelium) neovascularization using a modified 'treat and extend' dosing regimen of intravitreal antivascular endothelial growth factor therapy. Retina. 2010;30:1368-75.

50. Grossniklaus HE, Green WR. Choroidal neovascularization. Am J Ophthalmol. 2004;137:496-503.

51. Picton F, Muldrew KA, Reeves B, et al. Morphological features and determinants of new geographic atrophy developing in participants in the IVAN trial. Invest Ophthalmol Vis Sci. 2014;55:4977.

52. Willoughby AS, Ying GS, Toth CA, et al. Subretinal hyper-reflective material in the comparison of agerelated macular degeneration treatments trials. Ophthalmology. 2015;122(9):1846.e5-53.e5.

53. Lee JY, Folgar FA, Maguire MG, et al.. Outer retinal tubulation in the comparison of age-related macular degeneration treatments trials (CATT). Ophthalmology. 2014;121(12):2423-2431.

54. Hariri A, Nittala MG, Sadda SR. Outer retinal tubulation as a predictor of the enlargement amount of geographic atrophy in age-related macular degeneration. Ophthalmology. 2015;122(2):407-13.

55. Moussa K, Lee JY, Stinnett SS, Jaffe GJ. Spectral domain optical coherence tomography-determined morphologic predictors of age-related macular degeneration-associated geographic atrophy progression. Retina. 2013;33:1590-9.

56. Hariri MG, Nittala SR, Sadda. Outer retinal tubulation as a predictor of the enlargement amount of geographic atrophy in age-related macular degeneration. Ophthalmology. 2015;122:407-413

57. Ho J, Witkin AJ, Liu J, et al. Documentation of intraretinal retinal pigment epithelium migration via high-speed ultrahigh-resolution optical coherence tomography. Ophthalmology. 2011;118:687-93.

58. Schuman SG, Koreishi AF, Farsiu S, et al. Photoreceptor layer thinning over drusen in eyes with agerelated macular degeneration imaged in vivo with spectral-domain optical coherence tomography. Ophthalmology. 2009;116:488-96.

59. Folgar FA, Yuan EL, Sevilla MB, et al. Drusen volume and retinal pigment epithelium abnormal 
thinning volume predict 2-year progression of agerelated macular degeneration. Ophthalmology 2016;123(1):39e1-50e1.

60. Sleiman K, Veerappan M, Winter KP, et al. Optical coherence tomography predictors of risk for progression to non-neovascular atrophic age-related macular degeneration. Ophthalmology. 2017;124(12):1764-1777.

61. Kumar M, Mrejen S, Fung AT, Marsiglia M, Loh BK, Spaide RF. Retinal pigment epithelial cell loss assessed by fundus autofluorescence imaging in neovascular age-related macular degeneration. Ophthalmology. 2013;120(2):334-41.

62. Fleckenstein M, Wolf-Schnurrbusch U, Wolf S, von Strachwitz C, Holz FG, Schmitz-Valckenberg S. Imaging diagostics of geographic atrophy. Ophthalmologe. 2010;107(11):1007-155.

63. Göbel AP, Fleckenstein M, Schmitz-Valckenberg S, Brinkmann CK, Holz FG. Imaging geographic atrophy in age-related macular degeneration. Ophthalmologica. 2011;226(4):182-90. 